\title{
EXPLORANDO O TESTE AUDIT E SEUS PARÂMETROS EM UM HOSPITAL PEDIÁTRICO
}

Felipe Leonardo Rigo

Hospital Infantil João Paulo II / FHEMIG felipeleonardorigo@hotmail.com

Cassidy Tavares Silva

Hospital Infantil João Paulo II / FHEMIG

tavarescassidy@gmail.com

Mércia Beatriz Martins Silva Hospital Infantil João Paulo II / FHEMIG merciabiaII@gmail.com

Thaís Pereira Lopes de Souza Hospital Infantil João Paulo II / FHEMIG thaispls@live.com

\section{RESUMO}

INTRODUÇÃO: O consumo abusivo de bebidas alcoólicas constitui um dos principais problemas de saúde pública no mundo e anualmente 3 milhões de mortes são decorrentes do uso nocivo do álcool o que representa $5,3 \%$ das mortes mundiais. A recente pandemia causada pelo coronavírus 2019 (COVID-I9) e o isolamento social tem implicações que impactam no comportamento da saúde, o que inclui o consumo de álcool. OBJETIVO: Investigar o padrão de consumo de bebidas alcoólicas entre os profissionais de saúde durante a pandemia do COVID-19. METODOLOGIA: Estudo transversal, descritivo, quantitativo e realizado em um hospital público pediátrico da rede estadual de saúde e referência para o atendimento de doenças infecto parasitárias no estado de Minas Gerais. A coleta de dados foi realizada entre os meses de agosto a novembro de 2020. Para a coleta dos dados utilizou-se dois instrumentos, sendo o primeiro referente a questões do perfil sociodemográfico e ocupacional dos trabalhadores. Já o segundo instrumento foi o Alcohol Use DisordersIdentification Test (AUDIT). Estudo aprovado pelo Parecer n ${ }^{\circ}$ 4.I30.30I. RESULTADOS: Participaram do estudo 27I profissionais de saúde, sendo (91,2\%) mulheres, com idade entre 30 e 49 anos (67,9\%), auto declararam raça branca (39,6\%), solteiros (48,9\%), possuía religião $(87,4 \%)$, pós-graduação $(40 \%)$. Quanto as variáveis do padrão de consumo, $(65,3 \%)$ ingerem bebidas alcoólicas. Entre as bebidas mais consumidas observa-se a cerveja (35,6\%), seguido do vinho (27,5\%). A frequência de consumo foi de 2 a 4 vezes no mês em $(55,9 \%)$ e a cada consumo bebem em torno de I a 2 doses $(62,3 \%)$. Entre os principais motivos listados para o consumo de álcool temos, lazer/recreação (29,5\%), relaxar $(21,3 \%)$ e confinamento/tensão pela pandemia (5,I\%). Entre os participantes do estudo (I4,I\%) relataram que iniciaram ou tiveram aumento do consumo de bebidas alcoólicas durante a pandemia. Houve associação significativa para o uso de risco entre os entrevistados que tem familiares que consomem álcool $(\mathrm{p}<0,00 \mathrm{I})$ e entre os que bebiam antes da pandemia $(\mathrm{p}<0,00 \mathrm{I})$. CONCLUSÃO: $\mathrm{O}$ estudo evidenciou que a ingestão de bebidas alcoólicas entre os profissionais de saúde é frequente. É imperativo novas pesquisas que investigue o padrão de consumo de bebidas alcoólicas entre os profissionais de saúde. É fundamental que haja nas instituições de saúde políticas de ação com foco na promoção de hábitos saudáveis de vida.

PALAVRAS-CHAVE: Bebidas alcoólicas; Profissionais de Saúde; Pandemia COVIDI9 